\title{
Aller À La Rencontre D’hildegard De Bingen...
}

\section{Corinne Calvet Curbaille ${ }^{1}$}

\section{RÉSUMÉ}

Aller à la rencontre d'Hildegarde de Bingen, c'est aller à la découverte d'une singularité qui tente de se dire tout en s'extrayant par l'emploi d'une production, faisant œuvre multiple, de ce qu'elle nous annonce comme une certaine vérité sur sa jouissance féminine. II faudra revenir sur une production artistique peu commune brodée autour d'une rencontre avec un bout de réel. Elle va employer les éléments du savoir transmis et les mettre au service d'une cause divine, pour elle, et faire résonner pour nous ce qui de sa chair s'est épris de l'Autre dans cette lettre vivante et centrale au cœur de son écriture. L'hypothèse à ce travail se plait à interroger la jouissance féminine. "Cette jouissance qu'on éprouve et dont on ne sait rien, n'est-ce-pas ce qui nous met sur la voie de l'ex-sistence? Et, pourquoi ne pas interpréter une face de l'Autre, la face Dieu, comme supportée par la jouissance féminine? » (LACAN, 1975, p 71).

MOTS-CLÉS: Féminin, jouissances, création, objet voix, sinthome.

1 Psicóloga e Psicanalista. Trabalha há mais de 17 anos em um serviço de psiquiatria adulto no Centro Hospitalar de Béziers (França). Membro da Associação da Causa Freudiana na região francesa Languedoc Roussillon. Mestre desde 2013 em Pesquisa e Estudos Psicanalíticos e Estéticos pela Universidade Paul Valèry (França). Endereço de correspondência: 17 rue Henri Pégoud 34500 Béziers, France. Endereço eletrônico: curbaillecalvetc@aol.com 
" Il me semble que nous apprenions quelque chose de l'art, quand nous éprouvons ce que voudrait désigner le mot solitude » (BLANCHOT, 1955, p. 13)

"Le réel, dirai-je, c'est le mystère du corps parlant, c'est le mystère de l'inconscient. » (LACAN, 1975, p. 118)

C'est par les ondes, radiophoniques celles de « France Musique » que j'ai été initiée à la vie de ce personnage d'Hildegarde de Bingen. Quelques mots ont fait mouche pour moi qui suis intéressée par «Le génie féminin ». C'est ce titre que Julia Kristéva avait choisi pour nommer une série de 3 ouvrages dédiés à des personnalités fortes; Hannah Arendt, Mélanie Klein et Colette. Ouvrages dont la lecture est conseillée pour ceux et celles qui aiment les lectures éclairées.

Il était question dans cette émission d'histoire de la musique, de la singularité de cette femme qui fût l'une des premières à se lancer dans l'écriture musicale au $11^{\text {ème }}$ siècle. De nombreux éloges couraient à son sujet concernant son érudition, une voix décidée par une volonté de transmettre sa connaissance de l'homme, de la nature et de l'univers céleste qui lui ont fait dépeindre une véritable cosmologie. Une connaissance du domaine de l'homme et de la nature comme fruit de sa rencontre avec « le divin ».

Cette œuvre d'Hildegarde de Bingen fera l'objet dans une temporalité très éloignée d'elle de plusieurs siècles, d'une reconnaissance de ses pairs et lui vaudra une nomination émérite en 2012, celle de Docteur de l'église. Quatrième femme à qui aura été décerné ce titre honorifique après Catherine de Sienne, Sainte Thérèse d'Avila et de Thérèse de Lisieux.

Ce titre honorifique posthume vient nous rappeler quelques petites choses liées à la place des femmes au moyen âge.

En son temps, les femmes ne sont pas autorisées à dispenser un enseignement public. Rebecca Lenoir, à qui on doit une belle traduction des lettres d'Hildegarde de Bingen, nous le rappelle. Il semble que si Hildegarde de Bingen a pu transmettre « les fruits de sa passion » tant par une parole soutenue que par une volonté incessante d'écrire, c'est qu'elle a su très tôt qu'elle n'échapperait pas à l'autorité des pères de son église. Cette connaissance de l'ordre phallique de son monde lui a permis de s'entourer de pair mais aussi de demander une protection et une reconnaissance aux plus grands de son monde, comme Bernard de Clairvaux sous qui elle va placer "sa liberté de parole ». Elle peut poursuivre ainsi sa recherche divine tout en produisant les fruits de sa connaissance: ce savoir y faire révèle un choix existentiel orienté et nourri d'une grande solitude. Ce franchissement de la règle avec un certain savoir y 
faire avec les jouissances et le désir, étançons du sinthome lui permettra de mieux résister à des moments cruciaux de sa vie de bénédictine et de femme.

D'autre part, un autre nom lui sera donnée au $20^{\mathrm{ème}}$ siècle dans cette appellation de « mystique ». Ce nom de mystique n'est apparu qu'au $17^{\text {ème }}$ siècle. C'est plutôt au « mystère » qu'aurait été associé le nom de cette femme de son vivant. Ces deux noms, docteur de l'église et mystique, de l'après coup n'auront pas eu d'effets sur elle car elle n'aura pas eu le loisir de les entendre.

Mais ce terme de « mystique » nous intéresse quand même car c'est celui que choisit Lacan pour introduire une pluralité des jouissances.

Dans son séminaire «Encore » une Autre jouissance apparait qui n'est pas seulement liée à la sexualité où règne la loi phallique. Cette loi dite phallique est aussi celle qui fait de tous, hommes et femmes, des êtres parlant. Pour Lacan, c'est la rencontre entre la langue et le corps qui exile le sujet humain de la complétude sexuelle dont nous avons, dans l'éthologie, une représentation en une image fixe celle de l'instinct associé à la reproduction animale. Cette loi phallique vient rappeler l'impossible rapport sexuel mais aussi, médiatiser par le manque de l'objet adéquat, les relations amoureuses et désirantes entre les partenaires du couple.

Si cette loi phallique borde la rencontre pour le sujet avec le réel du partenaire sexuel, il persiste une part d'étrangeté et de différence - une part d'éprouvé - qui échappe à sa traduction. C'est ce reste qui vient en plus d'un manque pour certain sujet ouvrir un champ Autre à la jouissance. Non référée à la médiation sexuelle, cette Autre jouissance, n'entre pas dans le système des échanges signifiants, elle n'est pas pour tous et toutes, ce qui fera dire à Lacan (1975, p. 73) que, c'est du côté femme, de la bisexualité psychique (voir le tableau de la sexuation dans le séminaire «Encore ») qu'elle s'éprouve.

Pour une femme, déjà divisée par le signifiant, cette occurrence de jouissance la déporte en un lieu coupé d'elle-même -dans le sens de la représentation du soi - et ouvert sur une contigüité du passage que l'on pourrait comparer, par analogie seulement, aux règles Freudiennes en vigueur pour l'inconscient (pas de temporalité, pas de système d'opposition). Ces questions sont traitées très finement par une psychanalyste, Rose Paule Vinciguerra dans son ouvrage « Femmes Lacaniennes ». Si le féminin ne s'inscrit pas, s'il ne se transmet pas, par contre il ouvre à la créativité. En effet, il ouvre à une poétique vive du corps et de la langue et peut faire de l'imaginaire un allié pour traiter les maux du réel. 
Le signifiant, lui-même, s'il reste lié aux premières satisfactions de la création de la substance jouissante intraduisibles mais constitutives de ce premier impact dont j'ai parlé entre la langue et le corps, il est aussi le garde-fou du sujet. Selon le choix de structure du sujet, c'est-à-dire de son consentement ou non à se « laisser mordre » par le signifiant, en acceptant la perte irréductible qui lui est liée mais aussi en se laissant convoquer à des jouissances partielles et infimes voir hypothétiques, quelque chose d'une quête désirante pourra se dérouler et s'étayer de la chaine parlée. La structure existe donc toujours pour l'humain, qu'il ait accès à la parole ou qu'il soit en passe d'y parvenir, pour peu qui l'y consente. La singularité du sujet viendra en ponctuer le style et, de ce fait, il ne peut y avoir de hiérarchie de structure. II n'y a que des modes d'opérer différents face aux jouissances du réel, des modes symptomatiques de l'existence humaine. Toujours la structure donc, la névrose, la psychose et la perversion qui se décline mais avec un en plus qui pour certaine s'éprouve du reste. Ce reste, qui a des effets singuliers, opère pour le sujet qui s'y risque à un éprouvé d'une jouissance sans nom et sans loi. L'art du sujet s'y trouve convoqué, toute structure confondue, dans le retour, à se reconstituer comme corps jouissant sans aucun repère valide. Le non-sens du symbolique, la désorientation de l'imaginaire et la part du réel dévoilée oblige quelque part le sujet à s'y trouver avec ses propres limites. Livré donc à sa création intérieure - seule «Barrière contre le pacifique », pour citer un auteur, Marguerite Duras qui n'aura eu de cesse de s'y employer par une écriture hors norme, dont on ne pourra jamais dire qu'elle est de quelqu'un d'autre -, le sujet va s'employer, parfois avec le partenaire qu'il choisit son psychanalyste, à inventer sa propre solution de vie, son sinthome qui est aussi cette lettre dont Lacan parle, qui nous revient de l'Autre et dont on ne peut lire qu'une partie et se laisser éprouver dans sa lecture.

Ainsi, nous voyons que la psychanalyse, qu'elle soit « appliquée », c'est-à-dire celle qui se supporte de l'hypothèse du sujet de l'inconscient, en accroche les effets produits dans le monde des échanges sociaux (production de signifiants et d'objet hétéroclite) où qu'elle soit en « acte » pendant la cure où comme le dit Lacan « la psychanalyse est à réinventer », elle parle de la singularité de chacun et, pour nous, ici de cette rencontre avec cette femme, perdue pour notre siècle, mais qui n'a pas été avare dans ses tentatives de trouver des solutions et qui a laisser des traces de son symptôme de vivante. 
Ce personnage historique d'Hildegarde de Bingen va nous éclairer sur les divisions de sa subjectivité et surtout sur son originalité à traiter cette énigme pour une femme « d'être autre à elle-même ». II ne saurait être question d'embrasser la totalité de l'œuvre « Hildegarde de Bingen », mais plutôt de forcer un trait plutôt qu'un autre de sa formidable énergie de vouloir exister.

Nous pouvons dire que sa curiosité et son don prolifique pour la créativité laissent des témoignages majeurs de son passage sur cette terre. Une terre partagée avec nous certes mais une terre étrangère. N'étant pas historienne, je ne vais pas rentrer dans une exploration de ce temps, fort intéressant d'ailleurs, celui du moyen âge, mais plutôt me contenter de me référer à quelques écritures, les siennes et celles de Régine Pernoud en me laissant guider par ma propre lecture mais aussi en prenant un appui sur le film allemand «Visions » de Margarethe Von trotta.

Tout d'abord, quelques éléments biographiques et repères du quotidien de la vie de cette femme.

C'est donc au moyen Age, en 1098 que vient au monde Hildegarde de Bingen, en Allemagne du Sud-ouest.

A 8 ans elle est amenée par ses parents au monastère du Disibodenberg pour y être éduquée par la recluse Jutta Von Spanheim.

De la période de l'enfance plusieurs choses nous sont dites dans les témoignages d'Hildegarde.

D’abord, elle se présente comme ayant étant mise, dès 3 ans, en présence d'une source de clarté: « j'ai vu une telle lumière que mon âme en a été ébranlée, mais à cause de mon enfance, je n'ai rien pu en dire. »(PERNOUD, 2013, p. 14-15)

A 5 ans, « Vois donc le joli petit veau qui est dans cette vache. II est blanc avec des tâches au front, aux pieds et au dos. » (PERNOUD, 2013, p. 15) A sa naissance, le veau était tel qu'elle l'avait dit. Elle va se rendre compte que ses perceptions du monde restent étrangères aux autres, sa nourrice, ses parents, ses frères et sœurs (elle est la dixième enfant du ménage).

A 8 ans elle va « être donnée à Dieu » et baigner dans l'éducation religieuse. Dans le film «Vision » cette scène du don est traitée d'une façon exemplaire. L'enfant ne peut rien dire. Elle est déjà devenue la fille de sa mère de substitution. Juste un petit cri, « reste étouffé », de cette scène traumatique du départ de ses parents.

L'ordre monastique de Saint Benoit qui «prône la recherche de dieu » est devenu le quotidien de cette enfant, aux prises avec ses jouissances et une santé 
précaire ; « J'ai ignoré beaucoup de choses de la vie extérieure, car j'ai été souvent malade depuis le temps où ma mère m'allaitait et plus tard, ce qui nuisit à mon développement et m'empêcha de prendre des forces »(PERNOUD, 1995, p. 14).

A la majorité, après 12 ans, elle décide de prendre le voile. Et, selon la règle Bénédictine elle sera, elle aussi, une recluse comme l'a été sa mère de substitution.

A côté de l'enfermement qui est son quotidien, rythmé par la vie liturgique, elle traverse des périodes de maladies dont on ne sait rien et qui font dire à son biographe: «Parce que les vases d'argile s'éprouvent dans la fournaise et que le courage se perfectionne dans l'infirmité, les douleurs de santé ne lui manquèrent pas et se manifestèrent presque dès la petite enfance, nombreuses et quasi continuelles, de sorte que, dit- elle, elle se tenait rarement sur ses pieds » (PERNOUD, 1995, p. 17).

Elle reste sujette aux « éclairs du réel » ainsi qu'à ces moments où « le corps ne tient plus ». Elle va finir par parler de son vécu intime avec Jutta qui lui offrira la possibilité de se faire aider, conseiller, mais aussi concrètement d'être secondée dans l'écriture de ce vécu intime par un moine qui va l'accompagner de nombreuse année, Volmar. Une lettre écrite à Bernard de Clairvaux, nous renseigne sur les liens ténus entre la division subjective et les effets salvateurs d'une parole possible : Par moments, parce que je me tais sur ces visions, je reste terrassée sur mon lit par des lourdes infirmités qui m'empêchent de me lever »(DE BINGEN, 2007, p. 44).

A 38 ans environ, en 1136, Jutta meurt et les religieuses élisent Hildegarde comme abbesse. Dans le film, romancé certes, on voit d'un peu plus près la vie des recluses. Cette nomination d'abbesse est montrée dans le film. C'est Jutta qui lui demande d'abord de lui succéder. Hildegarde ne l'acceptera que soumise au vote de ses sœurs comme le veut la règle.

On ne sait pas grand-chose au fond de cette relation entre Jutta, cette mère « du savoir » et Hildegarde. Ce qu'il est relaté dans ses témoignages c'est sa grande curiosité qui on fait d'elle une élève attentive et douée. Est-ce pour romancer le film? Mais il y est question d'une autre sœur, amie d'Hildegarde, entrée jeune, elle aussi, au monastère qui voit dans cette nomination une part de l'amour convoité s'en aller vers une autre.

Ce savoir que lui transmet Jutta est un savoir y faire avec les moyens qui permettent de poursuivre une recherche « vers dieu ». 
Les biographes d'Hildegarde racontent qu'elle lui apprit les psaumes ainsi qu'à jouer du décacorde, l'instrument dont on s'accompagnait alors pour les chanter...toute éducation commence par le chant, et par le chant des psaumes ; « apprendre à lire » se dit « apprendre le psautier »... Jutta avait quelque peu négligé l'enseignement de la grammaire, portant son attention avant tout sur les textes eux-mêmes (PERNOUD, 1995, p. 16).

L'apprentissage de la langue liturgique est avant tout donc une pratique de la langue chantée. En fait, si le psautier est le même pour tous et toutes c'est la modulation de la voix qui va venir opérer une petite différence mais c'est surtout la jouissance de la langue qui va soutenir le souffle; les petites coupures, les tressautements, les envolées... Ce que l'on appelle aussi la tessiture de la voix. Ce mot de tessiture est intéressant car il nous ramène par sa définition au tissu. Cette définition nous donne quelque chose à entendre du côté de l'habillage du vocable interdit qui reste lui inaudible. La métaphore du tissu ouvre à celle du signifiant, du trou de structure et du maillage de la chaine parlée avec le symbolique, l'imaginaire et le réel. II est aussi une référence presque tactile au semblant qui noue identification et trait du réel. On parle aussi du « grain de voix » qui n'est pas sans rappeler le grain de la peau. La chair et le corps sont intimement liés.

Quelques mots peut-être pour éclairer cette pratique journalière rituelle et affirmée de cette langue chantée:

On sait à peu près comment se passe la vie des moniales bénédictines...leur journées est marquée par les heures canoniales...la nuit est interrompue par l'office de matines. Celles-ci sont chantées peu après minuit. Le moment de l'aurore, c'est-à-dire du lever du soleil, est pour moines et moniales celui du chant des laudes, qui suit l'office de prime (première heure) ; vient ensuite généralement la célébration de l'eucharistie, la messe, après laquelle dans la plupart des couvents a lieu le petit déjeuner ; puis vient l'office de tierce, dont le nom désigne la troisième heure après le lever du soleil ( 8 à 9heures selon les saisons), et un temps de travail jusqu'à l'heure de sexte (11h ou midi), que suit le repas. Le temps est libre ensuite jusqu'à l'heure de la none, la neuvième (généralement 14 ou 15 heures), où l'on reprend le travail, manuel ou intellectuel, collectif ou individuel ; l'heure de vêpres désigne l'office de fin de journée (18 à 19 heures), suivi du repas du soir et d'un temps libre, la récréation, généralement pris en commun. Souvent à lieu ensuite une réunion en chapitre, toutes les religieuses rassemblées en présence de l'abbesse; après quoi, au soleil couchant, c'est le dernier chant de l'office celui de complies; le silence doit alors régner pour permettre le repos de toutes. Au fil de ces diverses heures, l'ensemble du psautier-les cent cinquante psaumesaura été chanté dans l'ensemble de la semaine (PERNOUD, 1995, p. 17).

A 43 ans, en 1141, quelques années après la mort de Jutta, Hildegarde va entendre cet ordre auquel elle se pliera désormais au nom de la révélation qui lui a été faite sur le sens de sa vie; « Ecris ce que tu vois et ce que tu entends! » (DE BINGEN, 2011, p. 13). 
Dans les années qui suivirent il est a noté l'arrivée d'une jeune moniale, Richardis. Cette dernière va devenir la fille spirituelle d'Hildegarde de Bingen. Une affection profonde va les unir. La mère de Richardis, Marquise de Stade, va aider Hildegarde à s'installer avec les religieuses dans un nouveau lieu, voulu par l'abbesse, au grand dam des prêtres. Le film fait état d'une grossesse qui aurait suscité le choix, pour l'abbesse, d'éloigner du monastère des frères, les religieuses et vivre recluses entre femmes.

Durant les années qui s'écoulent de 1147 à 1150, les religieuses suivent l'abbesse dans leur nouvelle installation au Rupertsberg. Cette nouvelle implantation va susciter des critiques fortes intramuros ainsi que de la part des institutions cléricales.

De son fantasme venu tisser l'abandon de l'Autre à l'appel à « Un père », et de la place métaphorique du chant et de la parole musicale quelque chose nous en sera dit lors d'un événement qui va venir troubler sa construction de sa subjectivité.

Dans les années 1178 ses pairs vont lui refuser le droit de continuer à entretenir cette place quasiment « onirique » de sa pratique du chant dans la vie liturgique. II faut s'arrêter sur ce moment dans la mesure où nous avons vu que pour Hildegarde de Bingen, le chant, est comme une parole d'accueil du jour, de la nuit... de toutes ces infimes différences qui font aussi l'étrangeté du lien signifiant à l'Autre. Elle va devoir résister à « cette mise à mort subjective » afin de poursuivre sa route de chercheuse du divin en toutes choses.... L'interdit du chant est relatif à un épisode de sa vie de labeur de religieuse aux côtés des autres sans autre outil que sa foi.

Voilà ce que nous relate Rebecca Lenoir:

Le second conflit ne trouvera de solution qu'après la mort de Hildegarde. En 1178 , les prélats de Mayence frappent le monastère de l'interdiction de chanter les offices pour avoir accepté de donner une sépulture chrétienne à un jeune noble excommunié, qui, avant de mourir, se serait réconcilié avec l'Eglise, mais en privé et non publiquement. Les chanoines de Mayence réclame l'exhumation du corps, mais Hildegarde refuse catégoriquement: elle fait bénir la tombe et aplanir son emplacement pour qu'elle ne puisse être découverte. (DE BINGEN, 2007, p. 33)

Il n'est pas vain, ici, de rapprocher Hildegarde de Bingen d'une autre femme, celle-ci née avec la tragédie grecque, chez Sophocle, Antigone. C'est elle que Lacan choisie comme figure féminine pour nous orienter à la fin de son séminaire sur ce qu'est pour lui « L'éthique de la psychanalyse » et la relation qu'elle entretient avec le réel via le désir. «Antigone nous fait voir en effet le point de visée qui définit le 
désir...Cette image est pourtant au centre de la tragédie, puisque c'est celle, fascinante, d'Antigone elle -même »(LACAN, 1986, p. 290).

A la beauté du geste qui fait revenir et tient dans les bras d'une sépulture le corps d'un frère au sein de l'humaine condition, succède ce que Lacan vise dans l'ennoblissement du visage d'Antigone par ce trait de beauté. Cette fonction du beau, il va la placer au centre des enjeux pulsionnels d'où il énonce:

C'est dans la traversée de cette zone (qu'il relie plus haut à l'essence de la tragédie) que le rayon du désir se réfléchit et se rétracte à la fois, aboutissant à nous donner de cet effet si singulier, le plus profond, qui est l'effet du beau sur le désir... D'autre part, non réfracté, mais réfléchi, repoussé, son émoi, il le sait bien le plus réel. Mais là, il n'y a plus d'objet du tout (LACAN, 1986, p. 291).

Quelque chose de cette alchimie du vide et de la jouissance se trouvent à s'incarner chez Hildegarde ; comme un mouvement de bascule, une renégociation entre la vie et son au-delà; de l'établissement des règles, d'une conscience de l'autre... L'interdit du chant va perdurer pour elle mais elle n'aura de cesse de s'y confronter.

Ce qui nous intéresse dans ce lien avec ce séminaire de Lacan c'est le moment où tout en nous faisant apparaitre le visage d'Antigone, Lacan qui traitait peu de la musique fait référence alors à Aristote et déplie avec lui la fonction cathartique du passage par la musique et ses passions. "Mais tout le monde ne se met pas dans ces états d'enthousiasme...Ceux-là sont à portée d'être la proie d'autres passions, nommément la crainte et la pitié. Eh bien, à ceux-là aussi, une certaine musique, la musique, peut-on penser, qui est en cause dans la tragédie, où elle joue son rôle, apportera aussi une catharsis, un apaisement- par le plaisir et au-delà... » (LACAN, 1986, p. 288).

Hildegarde est donc réduite au silence. Mais elle va s'adresser aux maitres, les pères de son église par une demande de recours en grâce expliquant ses visions et sa mission divine quelle veut servir plus que tout au monde. Elle va l'obtenir auprès des grands personnages de la chrétienté, comme Bernard de Clairvaux et le pape luimême, Eugène III. Confortée dans ses actes et ses choix, cette reconnaissance du père et de ses pairs va s'étendre et elle va devenir une femme à qui peuvent s'adresser à leur tour, les grands de son monde et les autres. On va lui demander conseil en toute chose.

Son désir sera à nouveau contrarié car elle souhaite que Richardis reste auprès d'elle. En effet, elle vient d'être nommée abbesse d'un monastère de Saxe. Malgré les 
demandes incessantes d'Hildegarde de Bingen auprès des instances cléricales et auprès de la famille de Richardis, celle-ci partira mais mourra un an après son départ en 1151. Jusqu'à son départ, elle assistera l'abbesse dans ses travaux d'écritures en partageant sa tâche de secrétaire avec Volmar. Voici quelques phrases d'une lettre d'Hildegarde à Richardis; « J'aimais la noblesse de votre comportement, la sagesse et la pureté de votre âme et de tout votre être. »(PERNOUD, 1995, p. 56)

Un amour, qui se veut total donc, pour l'abbesse à l'adresse de sa fille spirituelle. Hildegarde bénédictine mais aussi femme n'échappe pas au ravage maternel.

En 1165, Hildegarde de Bingen crée un nouveau couvent de l'autre côté du Rhin, au-dessus de Rüdesheim, à Ei bingen. De ce lieu elle en portera le nom. En ce lieu elle est aussi inscrite comme bâtisseuse.

Hildegarde de Bingen n'aura cessé d'écrire, tel que cela lui fût demandé. Elle laisse une contribution à la liturgie chrétienne abondante et détaillée. Elle a produit le premier ouvrage de médecine européenne. Elle a aussi composé une œuvre musicale dense, et respectée aujourd'hui par les musicologues. Elle a entre autre chose écrit un opéra, «Ordo virtutum », à partir d'une de ses visions. "On y voit agir les Vertus, personnifiés, le Diable, l'âme fidèle qu'il tente de corrompre, l'Humilité, la Charité qui la défendent, etc. » (PERNOUD, 1995, p. 165).

Cette mise en scène des conflits psychiques qui étreignent tout autant la femme que la bénédictine est approchée dans le film de Margarethe Von trotta. On y voit les sœurs déguisées qui jouent la division subjective des sentiments sous le regard aiguisé et critique d'une visiteuse peu encline à soutenir ce projet. Ce projet, cette écriture, cette mise en scène, élèvent les sentiments humains au-delà de ce que peut entendre un certain ordre de la pratique religieuse. «La place de Dieu » ne doit pas être corrompue par le narcissisme d'une seule. Au fond ce que nous aimons depuis la psychanalyse Freudienne c'est pourtant ce qui se révèle comme voilée dans un projet d'existence toujours singulier ; un trait identificatoire, un ennoblissement du corps, une jouissance du processus créatif lui-même... L'assurance de la psychanalyse se résume à ce que déplie Lacan dans son séminaire sur l'éthique ; rien ne saurait recouvrir le manque de structure, ni l'objet, ni une idéologie abstraite, voir même une croyance celle en l'amour même. Même s'il faut attendre son dernier enseignement pour que s'épuise les tentatives de faire du parlêtre une consistance autre qu'un nouage (LACAN, 2005).

$51 \quad$ Psicanálise \& Barroco em revista | v.17, n. 01 | julho de 2019 
Hildegarde va poursuivre sa route de vivante. Elle va voyager et prendre la parole pour Dieu; cette « bouche de dieu » comme on appelle cette «prophète » en son pays.

Elle va échanger une correspondance soutenue avec les grands de son monde mais aussi avec d'autres femmes, comme elle, vivant la parole divine dans leur chair. Femme des échanges donc, qui n'est pas avide de parole et qui doit faire parler dieu.

Elle va mourir dans ce lieu qu'elle a créé en 1179 à l'âge de 81 ans.

Les éléments portés à la connaissance de sa vie éclairent nos consciences de la force d'une destinée hors du commun mais surtout d'une subjectivité qui s'est voulue prendre, à corps perdu tous les risques, menée par une «chose mystérieuse » dont elle nous dit qu' « Une lumière de feu, d'une extrême brillance, venant du ciel ouvert, fondit sur mon cerveau tout entier et tout mon corps, et toute ma poitrine comme une flamme qui cependant ne brulait pas, mais qui par sa chaleur enflammait de la façon que le soleil chauffe ce sur quoi il darde ses rayons. »(PERNOUD, 1995, p. 22)

A cet évènement de corps, qui tente de circonscrire quelques chose de cette première rencontre inaugurale constitutive, dans l'après-coup de cette morsure de lalangue fulgurante et morcellante, on peut associer, il me semble, ce qu'elle va dire, plus loin, de la mise en image et en mots, signifiante de ces premier moments. « Mais moi, poursuit Hildegarde, bien que j'aie vu et entendu cela...non par obstination, mais pour cause d'humilité, j'ai refusé d'écrire jusqu'à ce que j'aie été forcée sur le lit de douleurs où je suis tombée, atteinte d'un fléau de Dieu de telle façon qu'enfin j'ai été affligée de multiples infirmités...J'ai mis la main à l'écriture. » (PERNOUD, 1995, p. 24)

II faut se rappeler l'injonction surmoïque qui fait passer au tamis du langage cette lalangue première qui la somme « d'écrire et de dire ce qu'elle entend !», vis-àvis de laquelle nous voyons toute son ambivalence partagée, divisée qu'elle est entre la tentation de s'y soustraire « en corps » mais qui par le choix de son obéissance de structure l'amène à jouir phalliquement. La castration imposée par l'écriture et l'usage du dire passant par la parole lui permettent d'obtenir une pacification de cette chose mystérieuse, ce fléau de Dieu. Marguerite Duras a témoigné, elle aussi, de cette violence inouïe du choix de la structure qui ne peut nous apparaitre à nous que comme sublimé par l'art d'écrire.

De cette cause mystérieuse qu'elle nomme Dieu comme d'autre trouve un homme, une idéologie, réceptacle de ses projections cosmiques, elle va faire consister 
une approche du monde. Ce qui témoigne le plus de son élan narcissique c'est ce qui nous est livré certes par son « livre des œuvres divines » mais surtout par les dessins qui donnent une représentation de ce monde clos sur le mystère qui se veut rester entier: l'homme au centre de l'univers baignant dans toute la lumière divine y récupérant son éclat phallique. L'homme brillant! (PERNOUD, reproductions de dessins, 1995)

Ce qui pour moi nous rapproche un peu plus de cette jouissance Autre dont parle Lacan concernant les mystiques chez cette femme, c'est une façon de faire déjouer par l'écriture et le chant, la pesanteur de son adresse. Elle manie la légèreté musicale et l'art des voix entremêlées. Quelque chose me parait s'inverser à l'écoute (Sequentia, Le chant de l'extase).

C'est d'une certaine façon une jouissance du rythme, dans ces trajets, allers et retours, véritables coupures où la voix chantée se déplie dans le silence et dans ses formes de retour d'une voix devenue étrangère.

Cette petite fille restée sans voix à de nombreuses reprises, aux prises avec une jouissance localisée en son propre corps qui ne veut pas se soumettre à la loi et qui va baigner dans un univers chanté plutôt que parlé. Le chant est inclus bien sûr dans la parole, mais là, l'univers met en exergue cette occurrence qui accorde à la voix un lien pour la pratique quotidienne des corps. Ce rythme, devenu signifiant, s'inscrit dans un double mouvement, intérieur, extérieur et fait passer de la chair douloureuse à la mise en bouche du son. Mais le rituel ne vient pas recouvrir l'appel, seul indicatif de l'autre encore vivant.

II faut faire ici quelques rappels théoriques au sujet de cet objet dont Lacan parle dans son séminaire sur « L'angoisse ».

Dans la liste des objets partiels, c'est-à-dire, venant en creux donner une liste des lieux partiels et libidinaux du corps, passés au crible de l'Autre du désir -le sein, les fèces, le regard, la voix, le rien- certains sont plus à même de mettre en évidence la structure incomplète de l'Autre du désir.

II se trouve que la voix à la particularité de faire exister ce lieu de l'Autre du désir par son manque de réponse et donc par sa castration signifiante, qui rate à la nomination. Mais qui ouvre aussi dans une certaine démesure vers ce que Lacan dit au sujet de la castration de l'Autre «il n'y a pas d'Autre de l'Autre ». Même si Hildegarde s'emploie à faire résonner dieu c'est toujours de son manque dont il s'agit. 
Autrement dit, "La voix répond à ce qui se dit, mais elle ne peut pas en répondre. Autrement dit, pour qu'elle réponde, nous devons incorporer la voix comme l'altérité de ce qui se dit.»(LACAN, 2004, p. 318)

Le chant s'adresse à nul autre pareil, ce qui justifie qu'il ne s'arrête.

Pour conclure, j'aimerai terminer sur le témoignage de cette femme-chercheuse dans une lettre qu'elle adresse à Bernard de Clairvaux : «Moi, malheureuse et plus que malheureuse, en mon nom de femme depuis mon enfance, j'ai vu de grandes merveilles que ma langue ne peut proférer...moi qui jamais depuis mon enfance n'ai vécu en sécurité. » (PERNOUD, 1995, p. 76)

Le nom lui manquera toujours pour dire qui elle est, c'est ce défaut qui fait une femme.

De son défaut à l'ordre phallique elle connait, une femme, parfois, comme l'écrit très finement une psychanalyste, les détours par l'Autre jouissance. « Ça leur arrive disent les femmes quand elles en parlent, comme une folie. Si c'en est une, elle ne serait que passagère; ce serait plutôt une manière qu'ont les femmes de savoir-faire avec la folie, de s'en libérer. Rien n'en demeure cependant. Rien non plus ne s'en démontre-ni son contraire »(SOL APARICCIO, 1997, p 54). 


\section{RÉFÉRENCES BIBLIOGRAPHIQUES}

APARICIO, S. De la satisfaction, du bonheur et de l'extase, Revue de la Cause Freudienne, numéro 36, 1997, Paris, p 54

BLANCHOT, M. L'espace littéraire. Paris: Editions Gallimard, 1955.

DE BINGEN, H. Lettres. Grenoble: Editions Jérôme Millon, 2007.

DE BINGEN, H. Le livre des œuvres divines. Paris: Editions Albin Michel, 2011.

DURAS M. Un barrage contre le pacifique. Paris: Editions Gallimard, 1950.

DURAS M., Gauthier, X. Les Parleuses. Paris: Editions de Minuit, 2013.

KRISTEVA J. Le génie féminin, Hannah Arendt. Paris: Editions Gallimard, 1999.

LACAN, J. (1959-1960) Le séminaire livre VII, l'éthique de la psychanalyse. Paris: Editions du Seuil, Paris, 1986.

LACAN, J. (1962-1963) Le séminaire livre X, l'angoisse. Paris: Editions du Seuil, 2004.

LACAN, J. (1972-1973) Le séminaire livre XX, Encore. Paris: Editions du Seuil, 1975.

LACAN, J. (1975-1976) Le séminaire livre XXIII, Le sinthome. Editions du Seuil, 2005.

PERNOUD, R. Hildegarde de Bingen. Paris: Editions du Rocher, 1994.

VINCIGUERRA, R-P. Femmes Lacaniennes. Paris: Editions Michèle, 2014.

Musiques:

SEQUENTIA, Ordo Virtutum, Harmonia Mundi, BMG, 1998.

SEQUENTIA, Canticles of ecstasy, Harmonia Mundi, BMG, 1994.

Film:

VON TROTTA, M. (Direção). Vision”, 2009; mis sur Youtube le 26 Octobre 2015 par l'atelier de géobiologie. 


\section{Go To The Encounter Of Hildegard Von Bingen...}

\section{ABSTRACT}

To go to the encounter with Hildegard von Bingen is to go forwards the discovery of a singularity which tries to say itself as the same time that it extracts itself by the employment of a production, creating a multiple work, which announces us a certain truth of her feminine jouissance (an enjoyment beyond pleasure). It will be necessary to return to a rare artistic production embroidered around an encounter with a piece of real. She will employ the elements of the transmitted knowledge and put them in the service of this living and central letter in the heart of her writing. The hypothesis of this article pleases to interrogate the feminine jouissance. "This jouissance that we experience and that we do not know anything about, isn't it what put us in the path of the ex-sistence? And why not interpret one face of the Other, the face of God, as supported by the feminine jouissance?" (LACAN, 1975, p 71).

KEYWORDS: Feminine, jouissances, creation, object voice, sinthoma.La Voix Dans la Surdité 


\section{IR Ao Encontro De HiLdegard Von Bingen...}

\section{Resumo}

Ir ao encontro de Hildegard von Bingen é ir em direção da descoberta de uma singularidade que tenta se dizer ao mesmo tempo em que se extrai pela utilização de uma produção, fazendo obra múltipla, daquilo aue ela nos anuncia como uma certa verdade de seu gozo feminino. Será preciso retornar a uma produção artística pouco comum bordada em torno de um encontro com um pedaço de real. Ela utilisará os elementos do saber transmitido e os colocará a serviço de uma causa divina, para ela, que fará ressoar para nós aquilo que da sua carne é arrebatado pelo Outro nesta letra viva e central no coração de sua escrita. A hipótese deste trabalho se compraz a interrogar o gozo feminino. "Este gozo que se experimenta e sobre o qual nada se sabe, não é isso que nos coloca na via da ex-sistência? E por que não interpretar uma face do Outro, a face de Deus, como suporte do gozo feminino?" (LACAN, 1975, p 71).

PALAVRAS-CHAVE: Feminino, gozos, criação, objeto voz, sinthoma. 
Aller À La Rencontre D’hildegard De Bingen...

Recebido em: 15-09-2018

Aprovado em: 02-11-2018

(C) 2019 Psicanálise \& Barroco em revista

http://www.seer.unirio.br/index.php/psicanalise-barroco/index

revista@psicanaliseebarroco.pro.br

Programa de Pós-Graduação em Memória Social - UNIRIO

Memória, Subjetividade e Criação

www.memoriasocial.pro.br/proposta-area.php 